\title{
In silico tracking of SARS-CoV-2 Nsp1 structural variants in helix-turn-helix motif
}

\author{
Shokouh Rezaei ${ }^{1}$, Filipe Pereira ${ }^{2}$, and yahya sefidbakht ${ }^{3}$ \\ ${ }^{1}$ Affiliation not available \\ ${ }^{2}$ Centre for Functional Ecology, Department of Life Sciences, University of Coimbra, \\ Calçada Martim de Freitas, 3000-456 Coimbra, Portugal \\ ${ }^{3}$ Shahid Beheshti University
}

March 24, 2021

\begin{abstract}
SARS-CoV-2 non-structural protein 1 (Nsp1) is a virulence factor that inhibits the innate immune response and translation of host mRNAs. Despite the relevance of Nsp1, few studies have been conducted to understand the effect of mutations on Nsp1 structure and function. Here, we provide a molecular dynamics simulation of SARS-CoV-2 and SARS-CoV-1 Nsp1 conformational changes. Our data supports the idea that SARS-CoV-2 Nsp1 has a less compact structure than SARS-CoV-1 Nsp1. Moreover, several mutations in the helix-loop-helix motif of Nsp1 C-terminal that may affect the interactions of the Nsp1-ribosome complex were investigated. Disordered regions in Nsp1 probably affect host-virus interactions, cross-species transmission, and virus-host range. Overall, these findings reinforce the importance of studying Nsp1 conformational changes in new variants and its effect on virulence of SARS-CoV-2, by altering inhibition potency of host mRNA translation efficiency.
\end{abstract}

\section{In silico tracking of SARS-CoV-2 Nsp1 structural variants in helix-turn-helix motif}

Shokouh Rezaei ${ }^{1}$, Filipe Pereira ${ }^{2,3}$ and Yahya Sefidbakht ${ }^{*}, 1$

${ }^{1}$ Protein Research Center, Shahid Beheshti University, G.C., Velenjak, Tehran, Iran

${ }^{2}$ Centre for Functional Ecology, Department of Life Sciences, University of Coimbra, Calçada Martim de Freitas, 3000-456 Coimbra, Portugal

${ }^{3}$ IDENTIFICA genetic testing, Rua Simão Bolívar $2593^{\circ}$ Dir Tras. 4470-214 Maia, Portugal

* Author for Correspondence, Assistant Professor, Shahid Beheshti University, Tel: +98 21229905021 , Email:

y_sefidbakht@sbu.ac.ir

\section{Abstract}

SARS-CoV-2 non-structural protein 1 (Nsp1) is a virulence factor that inhibits the innate immune response and translation of host mRNAs. Despite the relevance of Nsp1, few studies have been conducted to understand the effect of mutations on Nsp1 structure and function. Here, we provide a molecular dynamics simulation of SARS-CoV-2 and SARS-CoV-1 Nsp1 conformational changes. Our data supports the idea that SARSCoV-2 Nsp1 has a less compact structure than SARS-CoV-1 Nsp1. Moreover, several mutations in the helixloop-helix motif of Nsp1 C-terminal that may affect the interactions of the Nsp1-ribosome complex were investigated. Disordered regions in Nsp1 probably affect host-virus interactions, cross-species transmission, and virus-host range. Overall, these findings reinforce the importance of studying Nsp1 conformational 
changes in new variants and its effect on virulence of SARS-CoV-2, by altering inhibition potency of host mRNA translation efficiency.

Key words: SARS-COV-2, Nsp1, Ribosome and Mutations

\section{Introduction}

The SARS-CoV-2 genome includes two large open reading frames (ORFs/ORF1ab) that occupy more than two-thirds of its length ${ }^{[1]}$. ORF1ab is proteolytically processed by two virally encoded proteinases (PLpro and $\mathrm{M}^{\mathrm{pro}}$ ) to produce 16 non-structural proteins (Nsp1 to Nsp16) ${ }^{[2,3]}$. Nsp1 is encoded by the gene closest to the 5 '-end of the genome, being also known as leader protein ${ }^{[4,5]}$. Nsp1 is believed to have identical physical features and biological activity among $\beta$-coronaviruses, although being poorly conserved ${ }^{[5,6]}$. Overall, Nsp1 has 180 amino acids (aa) forming two unstructured domains and one domain working as an interconnecting region between the two unstructured domains ${ }^{[7]}$. The crystal structure of SARS-CoV-2 Nsp1 revealed a six-stranded conformation ${ }^{[8]}$, with a C-terminal region holding an intrinsically disordered domain (residues 130-180) in comparison with its N-terminal region ${ }^{[9,10]}$. Based on previous studies, the interaction between the helical hairpin at the C-terminal domain of Nsp1 and the host 40S subunit of ribosome prohibits the entry of mRNA into the host ribosome ${ }^{[9]}$. Therefore, Nsp1 leads to translational shutoff of proteins from host mRNAs by binding the $40 \mathrm{~S}$ ribosome of the host cell ${ }^{[9,11,12]}$. The C-terminal domain of Nsp1 has two helices ${ }^{[11]}$ that are inserted into the entrance area of the ribosomal mRNA channel, probably contributing to the translational blockage ${ }^{[9,11]}$. In fact, Nsp1 leads to an endonucleolytic cleavage near the ${ }^{5}$ UTR of host mRNA (capped mRNA) when binding to ribosome ${ }^{[13]}$. Moreover, Nsp1 inhibits all cellular antiviral defense mechanisms such as Type I interferon (IFN-I) response, which leads to the shutoff of the response of the innate immune system ${ }^{[11,14]}$. Examination of the physical properties of the SARS-CoV-2 Nsp1 has shown that has a positive surface charge ${ }^{[15]}$. Despite the existence of a structural model of the Nsp1 ${ }^{[9,16]}$, few studied have been conducted on the dynamic aspects of this protein and how mutations may be change its conformation and function. Here, we used molecular dynamic (MD) and normal mode analysis (NMA) to further investigate the structure of Nsp1. We compared SARS-CoV-2 and SARS-CoV-1 Nsp1 and determined the effect of over 500 mutations on Nsp1 stability. These observations might highlight the relevance of understanding how mutations effect the Nsp1 inhibition of immune response and binding to the ribosome.

\section{Materials and methods}

\subsection{Nsp1 structural analysis using molecular dynamics (MD) simulation}

The Nsp1 protein sequences (Polyprotein1ab) from SARS-CoV-2 (P0DTD1) and SARS-CoV-1 (P0C6X7) were retrieved from the UniProtKB (http://www.uniprot.org/). The structure of the Nsp1-ribosome complex PDB was downloaded from SARS-CoV-2 Proteome-3D ${ }^{[17]}$ (https://sars3d.com/model/nsp1) to investigate the interaction between Nsp1 and the ribosomal 40S subunit.

The alignment between SARS-CoV-2 and SARS-CoV-1 Nsp1 sequences was performed using the EMBOSS Needleman-Wunsch method ${ }^{[18,19]}$. The I-TASSER server ${ }^{[20]}$ was used to build the 3D structure of both Nsp1 proteins. The best model was selected based on its C-score and the quality of the predicted structure was confirmed by PROCHECK ${ }^{[21,22]}$ and ProSA ${ }^{[23]}$, which calculate an overall quality score for 3D structures (Supplementary Figure S1). The local environment of amino acids was investigated through the WHAT IF coarse packing quality control ${ }^{[24]}$. The $\mathrm{H}++$ server was used to predict the protonation of histidine residues at $7.4 \mathrm{pH}{ }^{[25]}$. The UCSF Chimera (1.14) was used for visualization and analysis of structures ${ }^{[26]}$. The molecular dynamics (MD) simulation were carried out using the GROMACS 2020.2 software in OPLS force field. A water box was created with at least $1 \mathrm{~nm}(10 \AA)$ distances from the protein using the SPC water model and applying boundary conditions. The system neutralization was done by adding $\mathrm{Na}^{+}$and $\mathrm{Cl}^{-}$ions at the concentration of $0.1 \mathrm{M}$. The MD simulation was carried out to examine the quality of the model structures by investigating their stability via performing $100 \mathrm{~ns}$ simulations at a constant temperature $310 \mathrm{~K}$ (NVT). Energy minimization was performed in 50,000 steps to avoid any bad contacts generated while solvating the system. Then, the NPT optimization was done for $100 \mathrm{ps}$. To increase the likelihood 
of achieving the appropriate structure, the MD simulation was performed for $100 \mathrm{~ns}$ using the OPLS force field. The MD simulation was performed in three replicas for SARS-CoV-2 and two for SARS-CoV-1 Nsp1. At the end of this process, the GROMACS Tools package were used for the trajectory analysis; including root-mean-square deviation (RMSD), residue-based root mean square deviation (RMSF), radius of gyration $(\mathrm{Rg})$, solvent accessible surface areas (SASA), etc. The RMSD and Rg were also applied to calculate the Free Energy Surface (FES) of SARS-CoV-2 and SARS-CoV-1 Nsp1. The trajectory files of Principal Component Analysis (PCA) were analyzed by using g_covar and g_anaeig within the GROMACS package. Eigenvectors of the covariance matrices and the projections of the first two principal components were computed.

\subsection{Nsp1-ribosome complex and Residue interaction networks (RINs)}

PRODIGY web server ${ }^{[27]}$ was used to predict the binding affinity of Nsp1-ribosome complex from it 3D structure. The residue interaction networks (RINs) approach was used to investigate key residues of Nsp1 that could play a role in interaction with the ribosome. The network was computed and visualized by RING $2.0^{[28,29]}$ and Cytoscape, respectively. This network represents nodes (e.g. residues) and edges (e.g. bonds between the residue side chains), which are essential factors to network analysis.

\subsection{SARS-CoV-2 Nsp1 substitutions}

SARS-CoV-2 Nsp1 mutations (substitutions, insertions, and deletions) were retrieved from the Glu-CoV database (http://cov-glue.cvr.gla.ac.uk/) ${ }^{[30]}$. The identified substitutions in the Nsp1 C-terminal (helixturn-helix) were examined in the DynaMut ${ }^{[31]}$ web server to determine their effects on protein stability. The effect of mutations in protein stability and the estimation of change in the folding free energy upon the mutation $\left(\Delta \Delta \mathrm{G}^{\text {Destablizing }}\right)^{[31,32]}$ and vibrational entropy energy $\left(\Delta \Delta \mathrm{S}^{\mathrm{Vis}}\right)$ was predicted using the DynaMut tool ${ }^{[31]}$. MutaBind2 ${ }^{[33]}$ was used to evaluate the effects of mutations on Nsp1-ribosome interactions. This server computed changes in binding affinity upon single mutations $\left(\Delta \Delta \mathrm{G}^{\text {Binding }}\right)$.

\subsection{Nsp1 intrinsically disordered regions (IDRs)}

PONDR was used to investigate intrinsically disordered regions (IDRs) of SARS-CoV-2 Nsp1, which is based on several different predictors such as VL3 and VSL2 ${ }^{[34,35]}$. In each of these predictors, the first letter refers to the method of characterization (V: Various) and the second letter refers to the length of the disordered region [S: Short (8 - 9 residues), and L: Long (40 or more residues)].

\section{Results}

\subsection{Structural Analysis of MD Simulations}

Molecular dynamics (MD) simulations were performed for the Nsp1 of SARS-COV-2 and SARS-CoV-1. The RMSD plot indicates that both proteins reached equilibrium after $100 \mathrm{~ns}$ and that the SARS-CoV-1 Nsp1 deviation is less than the SARS-CoV-2 Nsp1. We also found that the Nsp1 F157S variant has less stability than the wild type Nsp1 (Figure 1a). The Nsp1 SARS-CoV-1 exhibited a lower Rg values compared to the SARS-CoV-2 Nsp1. The difference between $R_{g}$ of these two proteins is $\sim 0.12$, which indicates functional movements of SARS-CoV-2 Nsp1 due to the lesser compactness of the structure. In general, the Rg for both sequences showed a determined pattern: SARS-CoV-2 Nsp1 > SARS-CoV-1 Nsp1, as shown in Figure 1b. The RMS plot was constructed from the $100 \mathrm{~ns}$ data in order to understand the deviation of each Nsp1. The RMSF of the backbone for the SARS-CoV-2 Nsp1 displayed more flexible 164 to 170 residues (Lys, His, Ser, Ser, Gly, Val, and Ter), as compared to the SARS-CoV-1 Nsp1 (Figure 1c). This region corresponds to the C-terminal of the Nsp1 related to the protein interaction with the ribosome through CT, meaning that its flexibility could be related to its function.

In order to analyze H-bond interactions during the $100 \mathrm{~ns}$ simulation time, intermolecular H-bond plots were constructed for the Nsp1 of SARS-CoV-2 and SARS-CoV-1 (Figure 1d). We found that the SARSCoV-2 Nsp1 has an approximately similar number of intermolecular H-bonds as the SARS-CoV-1 Nsp1. In addition, the SASA analysis showed that the amino acid residues of both structures approximately exhibit a similar behavior of the hydrophilic and hydrophobic residues (Figure 1e). In order to understand the total 
motion of Nsp1 structures in the phase space, the first two eigenvectors were projected onto it. The results indicate that the motion features characterized by the two eigenvectors are different in the SARS-CoV-2 and SARS-CoV-1 Nsp1 proteins. As shown in Figure 2a, SARS-CoV-2 Nsp1 show a wider motions than SARS-CoV-1 Nsp1, indicating more conformational changes.

The plots in Figure 2b-c represent a comparative view of the Free Energy Surface (FES) landscape as a function of RMSD and Rg for Nsp1. The general trend applying to both Nsp1 proteins is that the higher the value of the two parameters (RMSD and $R g$ ), the lower the free energy surface. We found that the $R_{g}$ of SARS-CoV-2 Nsp1 is higher than SARS-CoV-1 Nsp1, suggesting that SARS-CoV-2 Nsp1 has less energy and more stability in compared with SARS-CoV-1 Nsp1.

\subsection{Structural analysis of Nsp1-ribosome complex and Residue interaction networks (RINs)}

The complex structure of the SARS-CoV-2 Nsp1 protein and human ribosomal 40S subunit were analyzed. The binding site of Nsp1 to the ribosome contains 26 residues at Nsp1 C-terminal (Supplementary Table S1). Among these residues, 17 residues form a helix-turn-helix (HTH) motif and lead to form Nsp1-ribosome complex. As shown in Table 1, HTH interacts simultaneously with different regions of the ribosome called "head region" including uS3 and "body region" including eS30 and uS5 (Figure 3). HTH interacts with uS3 by D152, E155, D156, E159, Q158, and N160 residues, as well as this motif involved in interaction with uS5 by Y154, F157, Q158, W161, T170, L173, L177, N178 residues. R175, E176, and G179 residues of HTH motif play a key role in forming Nsp1-ribosome (eS30) complex. We calculated the binding energy $\left(\Delta G^{\text {Binding }}\right)$ for these interactions, which showed that the interaction between the Nsp1 and uS3 is stronger than the other interactions (Table 2).

In addition, the key residues for Nsp1 binding the ribosome were determined by using stress and betweenness as the two main parameters of the local metrics. The analysis of Nsp1 (HTH motif) network revealed crucial residues including D156, F157, V169, T170, E172, L173, and M174 (Figure 4a). Moreover, the investigating betweenness and stress parameters for SARS-CoV-2 Nsp1 (HTH) binding the ribosome indicated E155, Q158, L173, M174, and N178 as residues of interest (Figure 4b). The high betweenness and stress values suggest that the node is a mediator of interactions with other nodes and that is probably a key structural residue. Therefore, we reported these amino acids as critical residues at the interface between the SARS-CoV-2 Nsp1 and ribosome, which may play a vital role in forming the complex.

\subsection{Effect of mutations on thermodynamics parameters and intrinsically disordered regions (IDRs)}

We identified 795 substitutions, 46 deletions and 3 insertion in SARS-CoV-2 Nsp1 genomic sequences. We found that 69 mutations occur in 17 major residues of the HTH motif identified by us. Among the 69 mutations, 11 mutations were found to occur in more than 10 sequenced SARS-CoV-2 genomes. We found that 6 out of 11 substitutions in the HTH motif had a $\Delta \Delta G^{\text {Destablizing }}<0$ (Figure 5a). Only one substitution (E159D) had a $\Delta \Delta G^{\text {Destablizing }}<-1$. On the contrary, substitutions such as M174K and T170I had $\Delta \Delta \mathrm{G}^{\text {Destablizing }}>1$. Overall, $\Delta \Delta \mathrm{G}$ values below zero suggest that the mutation causes destabilization of the protein; otherwise, it leads to stabilization in protein. We also found that some of these mutations also lead to changes in intra-molecular interactions: T170I and M174K, result in a new hydrophobic interaction; T170I removes a hydrogen bond; M174K forms a weak hydrogen bond, and M174K leads to form ionic interaction (Figure 5b). These results suggests that the changes in intra-molecular interactions may be responsible for the increased or decreased stability of protein.

The vibrational entropy energy $\left(\Delta \Delta \mathrm{S}^{\mathrm{Vib}}\right)$ analysis revealed differences between the wild and the mutated variants. The vibrational entropy provides an average of the configurational entropies of the protein structure in single minima of the energy landscape ${ }^{[36,37]}$. The $\Delta \Delta \mathrm{S}^{\mathrm{Vib}}<0$ of mutant type indicates the rigidity of the Nsp1 structure while $\Delta \Delta \mathrm{S}^{\mathrm{Vib}}>0$ shows the flexibility of the structure. As shown in Figure 5c, we found positive $\Delta \Delta \mathrm{S}^{\mathrm{Vib}}$ for R175H, L177F, L173P, E159K, and E159D mutations, suggesting they stabilize the Nsp1 structure. On the other hand, we found a most negative value of $\Delta \Delta S^{\mathrm{Vib}}$ for M174K ( -2.48$)$, suggesting that it may play a significant role in destabilizing the structure of Nsp1. Such mutations can 
increase or decrease the Nsp1 protein flexibility and affect the speed and efficiency of its interaction with the ribosome when occuring at the protein binding site.

In addition to 11 mutations, we identified W161 in the loop of HTH motif as causing a turn in that motif, and it leads to the helix disruption. This residue can also cause hydrophobic interactions (with F157) and stabilize the protein structure. Several mutations are known for this residue, including W161C, W161S, W161L, and W161R. Our study showed that W161C has $\Delta \Delta \mathrm{G}^{\text {Destablizing }}<-1$, which leads to instability of the protein structure. Mutation F157S can also affect the formation or loss of this hydrophobic interaction, which in turn reduces the stability of the Nsp1 in that region.

As shown in Figure $5 \mathrm{~d}$, changes in binding affinity $\left(\Delta \Delta \mathrm{G}^{\text {binding }}\right)$ due to point mutations for the 11 residues of the Nsp1 HTH motif were predicted. Positive values corresponded to N178S, R175H, L177F, M174K, L173P, N178K, F157L, and D156N (destabilizers) and negative values corresponded to T170I, E159K, and E159D (stabilizers), which led to decreased and increased binding affinity of Nsp1, respectively.

Finally, positions 120-180 of Nsp1 were identified as intrinsically disordered regions (IDRs), as determined by the VSL2 predictor (Figure 6). In line with this result, the PONDR VL3 predictor identified positions 150-180 (Figure 6), approximately related to the C-terminal protein. The SARS-CoV-2 Nsp1 positions 124152 form a loop contains G127, G130, G132, G133, G137, G146, and G150, which separates the HTH motif from the rest of the protein.

\section{Discussion}

The COVID-19 epidemic is spreading as an unsolvable problem worldwide. Despite the efforts of researchers, many details on SARS-CoV-2 biology remain unknown. Our study focused on the Nsp1 structure and stability by using different computational tools. We found that SARS-CoV-2 Nsp1 has a reduced compactness in comparison with SARS-CoV-1 Nsp1, with an increase in structural movements, suggesting this protein can more easily approach the active site of the ribosome compared to SARS-CoV-1 Nsp1. In addition, we found that the C-terminal of the SARS-CoV-2 Nsp1, in particular residues 164 to 170, are more flexible than other regions of SARS-CoV-2 Nsp1 and SARS-CoV-1 Nsp1, confirming the role of this region in the interaction with the $40 \mathrm{~S}$ subunit.

Interestingly, a previous study suggested that when SARS-CoV-2 5'-UTR bind to Nsp1 N-terminal, the covalently linked Nsp1 C-terminal is not able to bind the 40S subunit ${ }^{[38]}$. The authors suggest that this may be due to a steric factor in which Nsp1 C-terminal is unable to approach the 40S subunit to form the Nsp1-ribosome complex. We found that mutation R24C had the highest frequency among all protein substitution mutations identified by us. Therefore, the occurrence of this mutation in the Nsp1 N-terminal may change the affinity of Nsp1 binding to the 5'-UTR of SARS-CoV-2, perhaps making it more effective in using the host ribosome for translation of viral proteins.

The Nps1 C-terminal was identified as being intrinsically disordered. In addition, E155, F157, D156, Q158, V169, T170, L173, F172, M174, and N178 emerged from the RIN analysis of betweenness and stress as 10 key residues governing the stability and affinity of the Nsp1 for interaction with ribosome. We also found that 11 mutations at HTH motif, which affect the Nsp1 structure, stability and binding affinity. Structural studies revealed that IDRs influence viral genome (RNA) adaptive capacity, host-virus interactions, virushost range, cross-species transmission, and host tropism ${ }^{[39-43]}$. In antigen selection, IDRs may induce an undesirable immune response, such as weak or even completely non-immune responses ${ }^{[44]}$. Thus, these results indicated that IDRs may affect the interaction between the Nsp1 and the ribosome. If Nsp1 is used as an antigen, it may lead to an inappropriate immune response due to the presence of CT IDRs.

\section{Conclusion}

Among 795 Nsp1 substitutions, 69 belonged to the HTH motif in the C-terminal region. In this article, out of 69 mutations, only 11 substitutions with a frequency above 10 were examined and it was revealed that these mutations affect the thermodynamic parameters of the interaction between the protein and the ribosome. The effect of HTH mutations may increase the pathogenicity of SARS-CoV-2 and in turn, increase the inhibition 
of host protein translation. Therefore, findings suggest that Nsp1 should be considered as an important virulence factor because more mutations in the binding motif of Nsp1 may have drastic consequences.

\section{Acknowledgement}

The support and resources from the Center for High Performance Computing at Shahid Beheshti University of Iran are gratefully acknowledged. We would also like to thank all the researches who have kindly shared genomes on public databases.

\section{Funding}

This research has been conducted using the research credits of Shahid Beheshti University, G.C., Tehran, Iran (Grant n. sad/600/1451). This work was partially supported by the Fundação para a Ciência e a Tecnologia [RESEARCH 4 COVID-19 project n. 029] and the EOSCsecretariat.eu COVID-19 Fast Track Funding. EOSCsecretariat.eu has received funding from the European Union's Horizon Programme call H2020-INFRAEOSC-05-2018-2019, grant Agreement number 831644.

\section{References}

1. Gordon, D. E., Jang, G. M., Bouhaddou, M., Xu, J., Obernier, K., White, K. M., O’Meara, M. J., Rezelj, V. V., Guo, J. Z., Swaney, D. L., Tummino, T. A., Hüttenhain, R., Kaake, R. M., Richards, A. L., Tutuncuoglu, B., Foussard, H., Batra, J., Haas, K., Modak, M., ... Krogan, N. J. (2020). A SARS-CoV-2 protein interaction map reveals targets for drug repurposing. Nature , 583 (7816), 459-468. https://doi.org/10.1038/s41586-020$2286-9$

2. Clark, L. K., Green, T. J., \& Petit, C. M. (2020). Structure of Nonstructural Protein 1 from SARS-CoV-2. Journal of Virology ,95 (4). https://doi.org/10.1128/jvi.02019-20

3. Egorova, T., \& Alkalaeva, E. (2020). Nsp1 of SARS-CoV-2 Stimulates Host Translation Termination .

4. Kamitani, W., Narayanan, K., Huang, C., Lokugamage, K., Ikegami, T., Ito, N., Kubo, H., \& Makino, S. (2006). Severe acute respiratory syndrome coronavirus nsp1 protein suppresses host gene expression by promoting host mRNA degradation. Proceedings of the National Academy of Sciences of the United States of America , 103 (34), 12885-12890. https://doi.org/10.1073/pnas.0603144103

5. Vankadari, N., Jeyasankar, N. N., \& Lopes, W. J. (2020). Structure of the SARS-CoV-2 Nsp1/5'Untranslated Region Complex and Implications for Potential Therapeutic Targets, a Vaccine, and Virulence. The Journal of Physical Chemistry Letters , 9659-9668. https://doi.org/10.1021/acs.jpclett.0c02818

6. Tohya, Y., Narayanan, K., Kamitani, W., Huang, C., Lokugamage, K., \& Makino, S. (2009). Suppression of Host Gene Expression by nsp1 Proteins of Group 2 Bat Coronaviruses. Journal of Virology , 83 (10), 5282-5288. https://doi.org/10.1128/jvi.02485-08

7. Pandala, N., Cole, C. A., McFarland, D., Nag, A., \& Valafar, H. (2020). A Preliminary Investigation in the Molecular Basis of Host Shutoff Mechanism in SARS-CoV. Proceedings of the 11th ACM International Conference on Bioinformatics, Computational Biology and Health Informatics, BCB 2020, August . https://doi.org/10.1145/3388440.3412483

8. Semper, C., Watanabe, N., \& Savchenko, A. (2021). Structural characterization of nonstructural protein 1 from SARS-CoV-2.IScience, 24 (1), 101903. https://doi.org/10.1016/j.isci.2020.101903

9. Schubert, K., Karousis, E. D., Jomaa, A., Scaiola, A., Echeverria, B., Gurzeler, L. A., Leibundgut, M., Thiel, V., Muhlemann, O., \& Ban, N. (2020). SARS-CoV-2 Nsp1 binds the ribosomal mRNA channel to inhibit translation. Nature Structural and Molecular Biology ,27 (10), 959-966. https://doi.org/10.1038/s41594-020-0511-8

10. Kumar, A., Kumar, A., Kumar, P., Garg, N., \& Giri, R. (2020). SARS-CoV-2 NSP1 C-terminal region (residues 130-180) is an intrinsically disordered region. BioRxiv . https://doi.org/10.1101/2020.09.10.290932 
11. Thoms, M., Buschauer, R., Ameismeier, M., Koepke, L., Denk, T., Hirschenberger, M., Kratzat, H., Hayn, M., MacKens-Kiani, T., Cheng, J., Straub, J. H., Sturzel, C. M., Frohlich, T., Berninghausen, O., Becker, T., Kirchhoff, F., Sparrer, K. M. J., \& Beckmann, R. (2020). Structural basis for translational shutdown and immune evasion by the Nsp1 protein of SARS-CoV-2. Science , 369 (6508), 1249-1256. https://doi.org/10.1126/SCIENCE.ABC8665

12. Lokugamage, K. G., Narayanan, K., Huang, C., \& Makino, S. (2012). Severe Acute Respiratory Syndrome Coronavirus Protein nsp1 Is a Novel Eukaryotic Translation Inhibitor That Represses Multiple Steps of Translation Initiation. Journal of Virology , 86 (24), 13598-13608. https://doi.org/10.1128/jvi.01958-12

13. Tanaka, T., Kamitani, W., DeDiego, M. L., Enjuanes, L., \& Matsuura, Y. (2012). Severe Acute Respiratory Syndrome Coronavirus nsp1 Facilitates Efficient Propagation in Cells through a Specific Translational Shutoff of Host mRNA. Journal of Virology ,86 (20), 11128-11137. https://doi.org/10.1128/jvi.01700-12

14. Bouayad, A. (2020). Innate immune evasion by SARS-CoV-2: Comparison with SARS-CoV. Reviews in Medical Virology , 30 (6), 1-9. https://doi.org/10.1002/rmv.2135

15. Vankadari, N., Jeyasankar, N. N., \& Lopes, W. J. (2020). Structure of the SARSCoV-2 Nsp1/5'-Untranslated Region Complex and Implications for Potential Therapeutic Targets, a Vaccine, and Virulence.Journal of Physical Chemistry Letters , 11 (22), 9659-9668. https://doi.org/10.1021/acs.jpclett.0c02818

16. Almeida, M. S., Johnson, M. A., Herrmann, T., Geralt, M., \& Wuthrich, K. (2007). Novel $\beta$-Barrel Fold in the Nuclear Magnetic Resonance Structure of the Replicase Nonstructural Protein 1 from the Severe Acute Respiratory Syndrome Coronavirus. Journal of Virology , 81 (7), 3151-3161. https://doi.org/10.1128/jvi.01939-06

17. Alsulami, A. F., Thomas, S. E., Jamasb, A. R., Beaudoin, C. A., Moghul, I., Bannerman, B., Copoiu, L., Vedithi, S. C., Torres, P., \& Blundell, T. L. (2021). SARS-CoV-2 3D database: understanding the coronavirus proteome and evaluating possible drug targets.Briefings in Bioinformatics , 00 (September 2020), 1-12. https://doi.org/10.1093/bib/bbaa404

18. Needleman, S. B., \& Wunsch, C. D. (1970). A general method applicable to the search for similarities in the amino acid sequence of two proteins. Journal of Molecular Biology , 48 (3), 443-453. https://doi.org/10.1016/0022-2836(70)90057-4

19. Rice, P., Longden, L., \& Bleasby, A. (2000). EMBOSS: The European Molecular Biology Open Software Suite. Trends in Genetics ,16 (6), 276-277. https://doi.org/10.1016/S0168-9525(00)02024-2

20. Yang, J., \& Zhang, Y. (2015). I-TASSER server: New development for protein structure and function predictions. Nucleic Acids Research , 43 (W1), W174-W181. https://doi.org/10.1093/nar/gkv342

21. Laskowski, R. A., MacArthur, M. W., Moss, D. S., \& Thornton, J. M. (1993). PROCHECK: a program to check the stereochemical quality of protein structures. Journal of Applied Crystallography ,26 (2), 283-291. https://doi.org/10.1107/s0021889892009944

22. Laskowski, R. A., Rullmann, J. A. C., MacArthur, M. W., Kaptein, R., \& Thornton, J. M. (1996). AQUA and PROCHECK-NMR: Programs for checking the quality of protein structures solved by NMR. Journal of Biomolecular NMR , 8 (4), 477-486. https://doi.org/10.1007/BF00228148

23. Wiederstein, M., \& Sippl, M. J. (2007). ProSA-web: Interactive web service for the recognition of errors in three-dimensional structures of proteins. Nucleic Acids Research , 35 (SUPPL.2), 407-410. https://doi.org/10.1093/nar/gkm290

24. Vriend, G., \& Sander, C. (1993). Quality control of protein models: Directional atomic contact analysis. Journal of Applied Crystallography , 26 (pt 1), 47-60. https://doi.org/10.1107/S0021889892008240 
25. Anandakrishnan, R., Aguilar, B., \& Onufriev, A. V. (2012). H++ 3.0: Automating pK prediction and the preparation of biomolecular structures for atomistic molecular modeling and simulations. Nucleic Acids Research , 40 (W1), 537-541. https://doi.org/10.1093/nar/gks375

26. Pettersen, E. F., Goddard, T. D., Huang, C. C., Couch, G. S., Greenblatt, D. M., Meng, E. C., \& Ferrin, T. E. (2004). UCSF Chimera - A visualization system for exploratory research and analysis. Journal of Computational Chemistry , 25 (13), 1605-1612. https://doi.org/10.1002/jcc.20084

27. Xue, L. C., Rodrigues, J. P., Kastritis, P. L., Bonvin, A. M., \& Vangone, A. (2016). PRODIGY: A web server for predicting the binding affinity of protein-protein complexes. Bioinformatics ,32 (23), 3676-3678. https://doi.org/10.1093/bioinformatics/btw514

28. Martin, A. J. M., Vidotto, M., Boscariol, F., Di Domenico, T., Walsh, I., \& Tosatto, S. C. E. (2011). RING: networking interacting residues, evolutionary information and energetics in protein structures.Bioinformatics , 27 (14), 2003-2005. https://doi.org/10.1093/bioinformatics/btr191

29. Piovesan, D., Minervini, G., \& Tosatto, S. C. E. (2016). The RING 2 . 0 web server for high quality residue interaction networks .44 (May), 367-374. https://doi.org/10.1093/nar/gkw315

30. Singer, J., Gifford, R., Cotten, M., \& Robertson, D. (2020). CoV-GLUE: A Web Application for Tracking SARS-CoV-2 Genomic Variation.Preprints , June , 2020060225. https://doi.org/10.20944/preprints202006.0225.v1

31. Rodrigues, C. H. M., Pires, D. E. V, \& Ascher, D. B. (2018).DynaMut : predicting the impact of mutations on protein conformation, flexibility and stability . 46 (April), 350-355. https://doi.org/10.1093/nar/gky300

32. Rezaei, S., Sefidbakht, Y., \& Uskoković, V. (2020). Comparative molecular dynamics study of the receptor-binding domains in SARS-CoV-2 and SARS- CoV and the effects of mutations on the binding affinity. Journal of Biomolecular Structure and Dynamics , 0 (0), 1-20. https://doi.org/10.1080/07391102.2020.1860829

33. Zhang, N., Chen, Y., Lu, H., Zhao, F., Alvarez, R. V., Goncearenco, A., Panchenko, A. R., \& Li, M. (2020). MutaBind2: Predicting the Impacts of Single and Multiple Mutations on Protein-Protein Interactions. IScience, 23 (3), 100939. https://doi.org/10.1016/j.isci.2020.100939

34. Lieutaud, P. (2016). How disordered is my protein and what is its disorder for? A guide through the " dark side " of the protein universe . 4 (1), 1-33. https://doi.org/10.1080/21690707.2016.1259708

35. Vinterhalter, G., Kova, J. J., Uversky, V. N., \& Pavlovi, G. M. (2021). International Journal of Biological Macromolecules Bioinformatics analysis of correlation between protein function and intrinsic disorder . 167 , 446-456. https://doi.org/10.1016/j.ijbiomac.2020.11.211

36. Chand, G. B., Banerjee, A., \& Azad, G. K. (2020). Identification of novel mutations in RNA-dependent RNA polymerases of SARS-CoV-2 and their implications on its protein structure. PeerJ , 2020 (7), 1-11. https://doi.org/10.7717/peerj.9492

37. Goethe, M., Fita, I., \& Rubi, J. M. (2015). Vibrational entropy of a protein: Large differences between distinct conformations. Journal of Chemical Theory and Computation , 11 (1), 351-359. https://doi.org/10.1021/ct500696p

38. Shi, M., Wang, L., Fontana, P., Vora, S., Zhang, Y., Fu, T. M., Lieberman, J., \& Wu, H. (2020). SARS-CoV-2 Nsp1 suppresses host but not viral translation through a bipartite mechanism. BioRxiv ,2 , 1-16. https://doi.org/10.1101/2020.09.18.302901

39. Charon, J., Barra, A., Walter, J., Millot, P., Hébrard, E., Moury, B., \& Michon, T. (2018). First Experimental Assessment of Protein Intrinsic Disorder Involvement in an RNA Virus Natural Adaptive Process.Molecular Biology and Evolution , 35 (1), 38-49. https://doi.org/10.1093/molbev/msx249 
40. Walter, J., Charon, J., Hu, Y., Lachat, J., Leger, T., Lafforgue, G., Barra, A., \& Michon, T. (2019). Comparative analysis of mutational robustness of the intrinsically disordered viral protein $\mathrm{VPg}$ and of its interactor eIF4E. PLoS ONE , 14 (2), 1-13. https://doi.org/10.1371/journal.pone.0211725

41. Mozzi, A., Forni, D., Cagliani, R., Clerici, M., Pozzoli, U., \& Sironi, M. (2020). Intrinsically disordered regions are abundant in simplexvirus proteomes and display signatures of positive selection. Virus Evolution , 6 (1), 1-12. https://doi.org/10.1093/ve/veaa028

42. Barik, S. (2020). Genus-specific pattern of intrinsically disordered central regions in the nucleocapsid protein of coronaviruses. Computational and Structural Biotechnology Journal , 18 , 1884-1890. https://doi.org/10.1016/j.csbj.2020.07.005

43. Sen, S., Dey, A., Bandhyopadhyay, S., \& Uversky, V. N. (2012). Understanding Structural Malleability of the SARS-CoV-2 Proteins and their Relation to the Comorbidities SARS-CoV-2 . 774 , 1-17.

44. Macraild, C. A., Richards, J. S., Anders, R. F., \& Norton, R. S. (2016). Antibody Recognition of Disordered Antigens. Structure ,24 (1), 148-157. https://doi.org/10.1016/j.str.2015.10.028

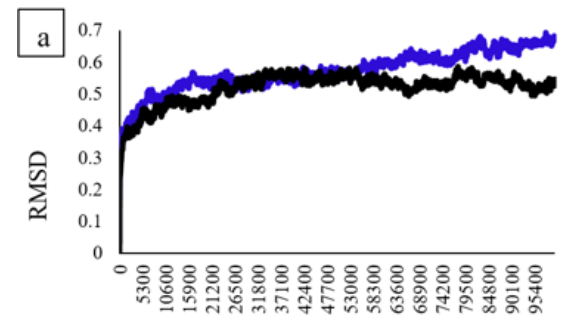

Time (ps)

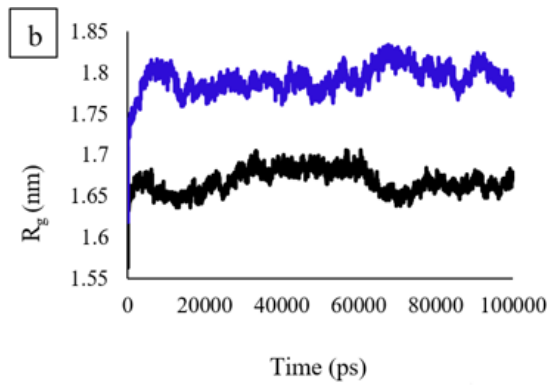

Time (ps)
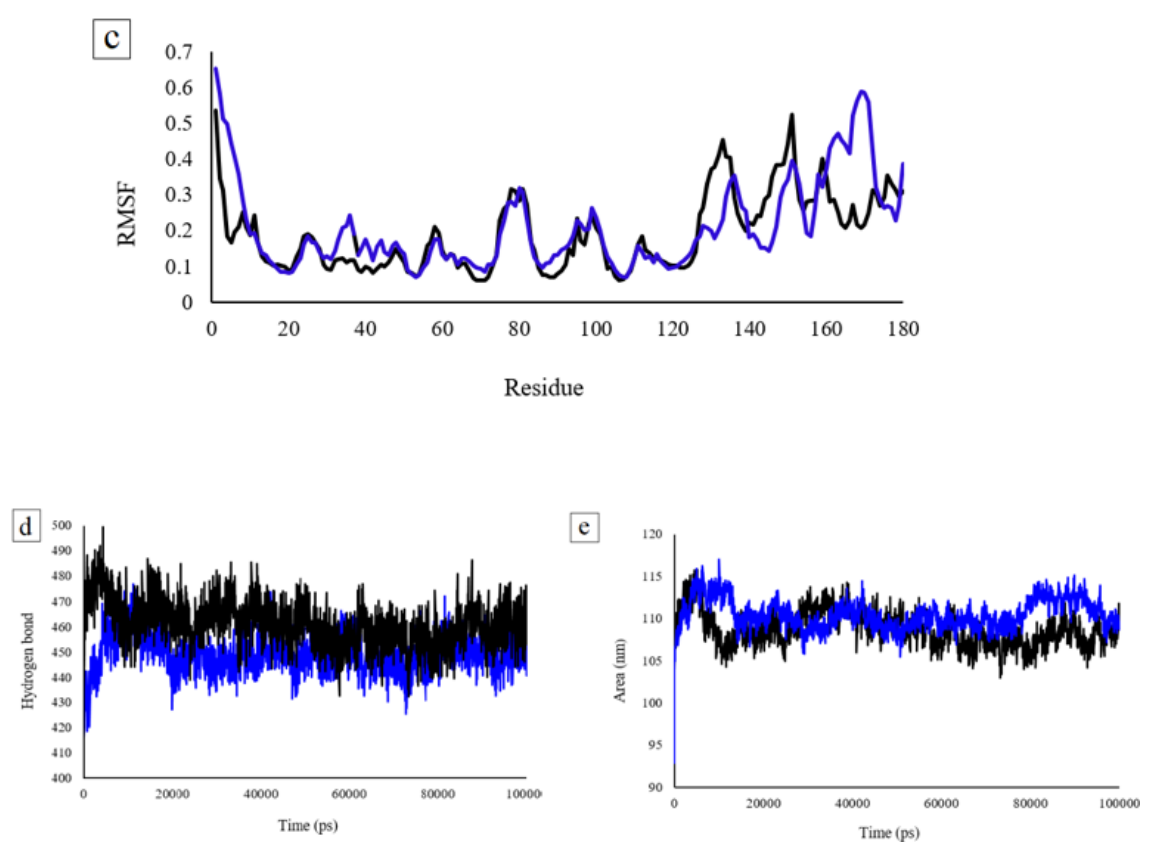
$\mathrm{a}$
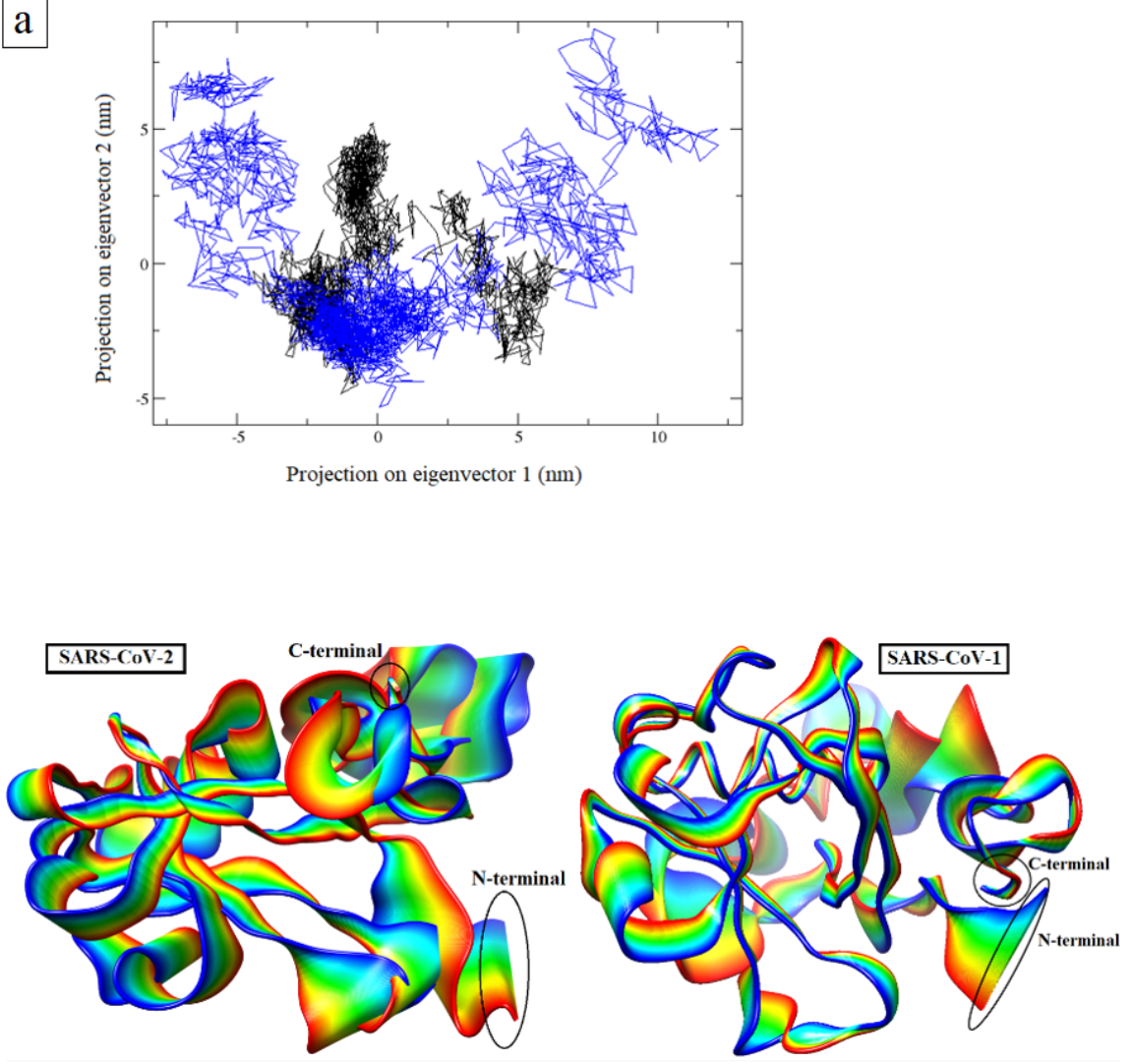

b
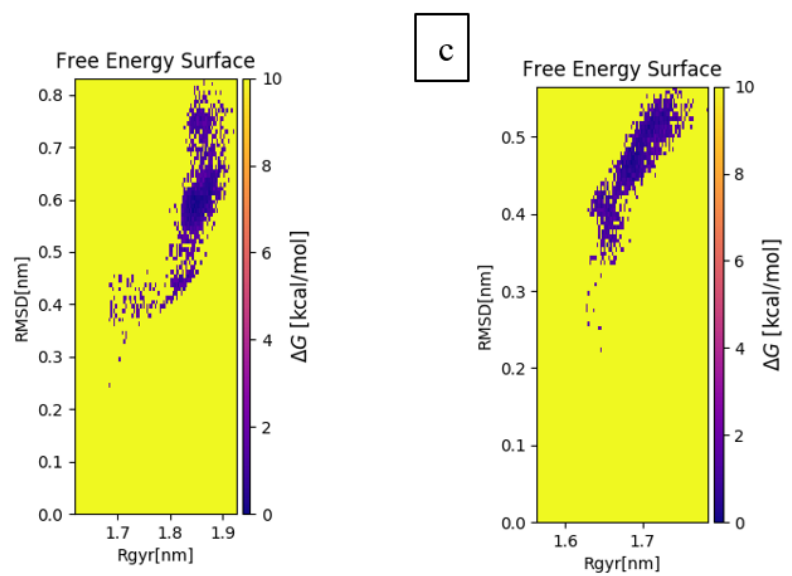


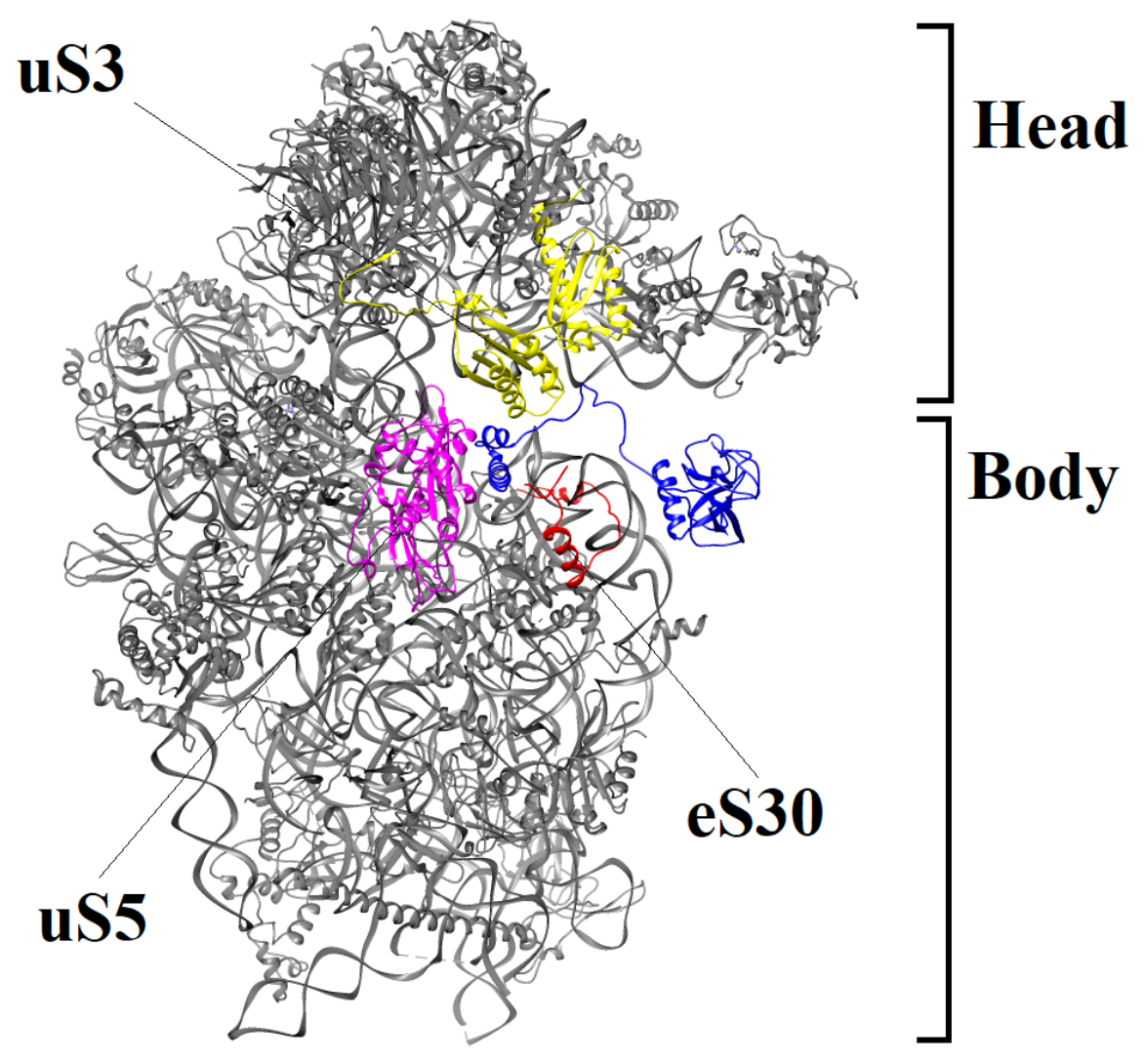




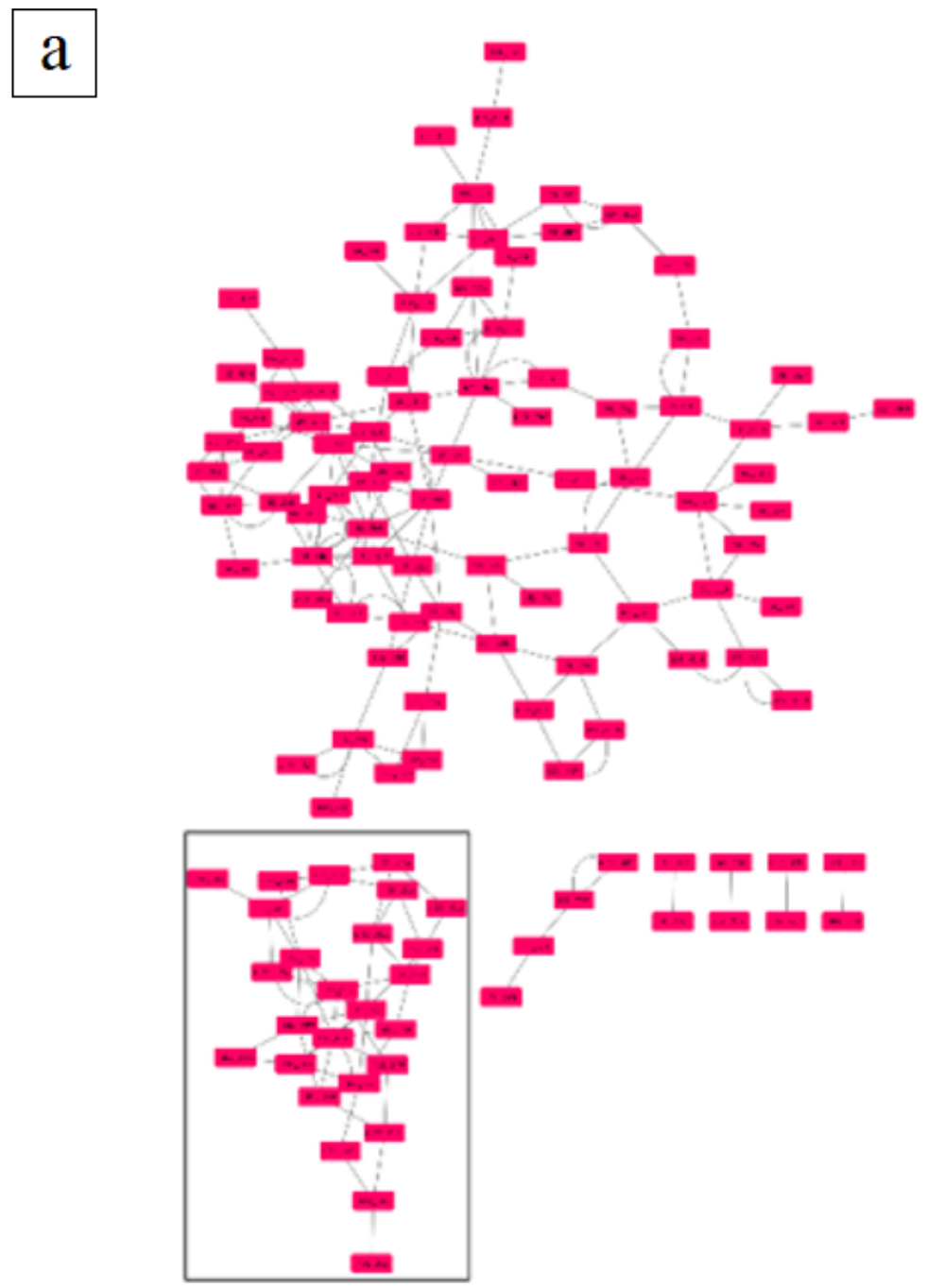

b

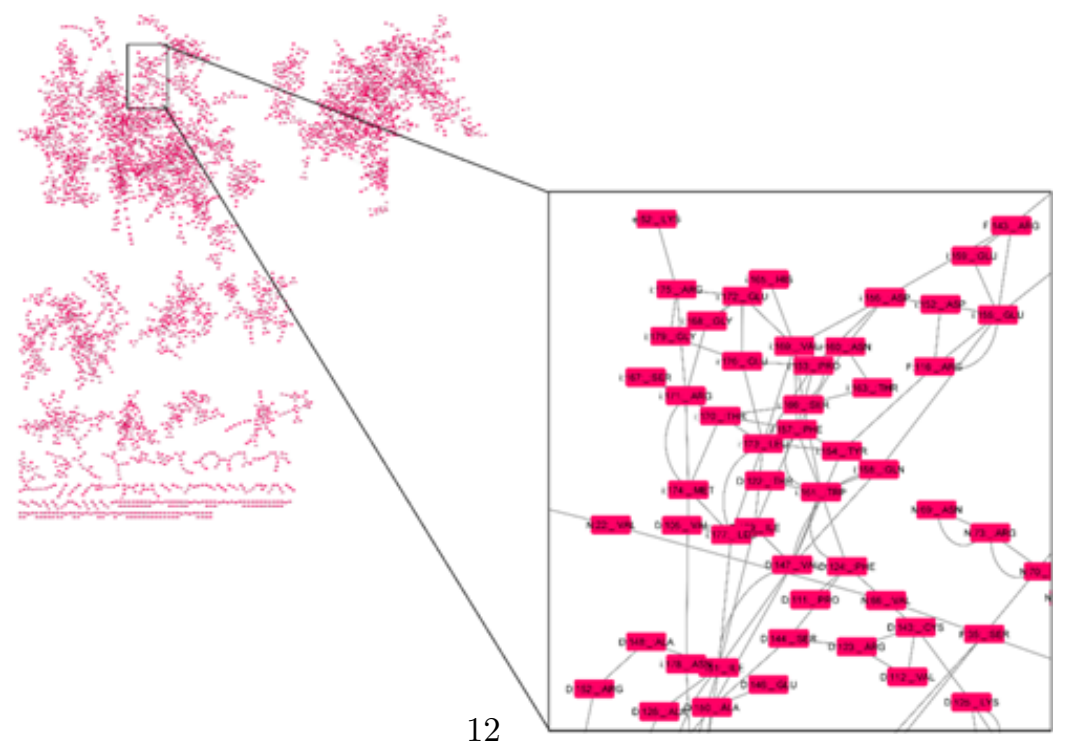



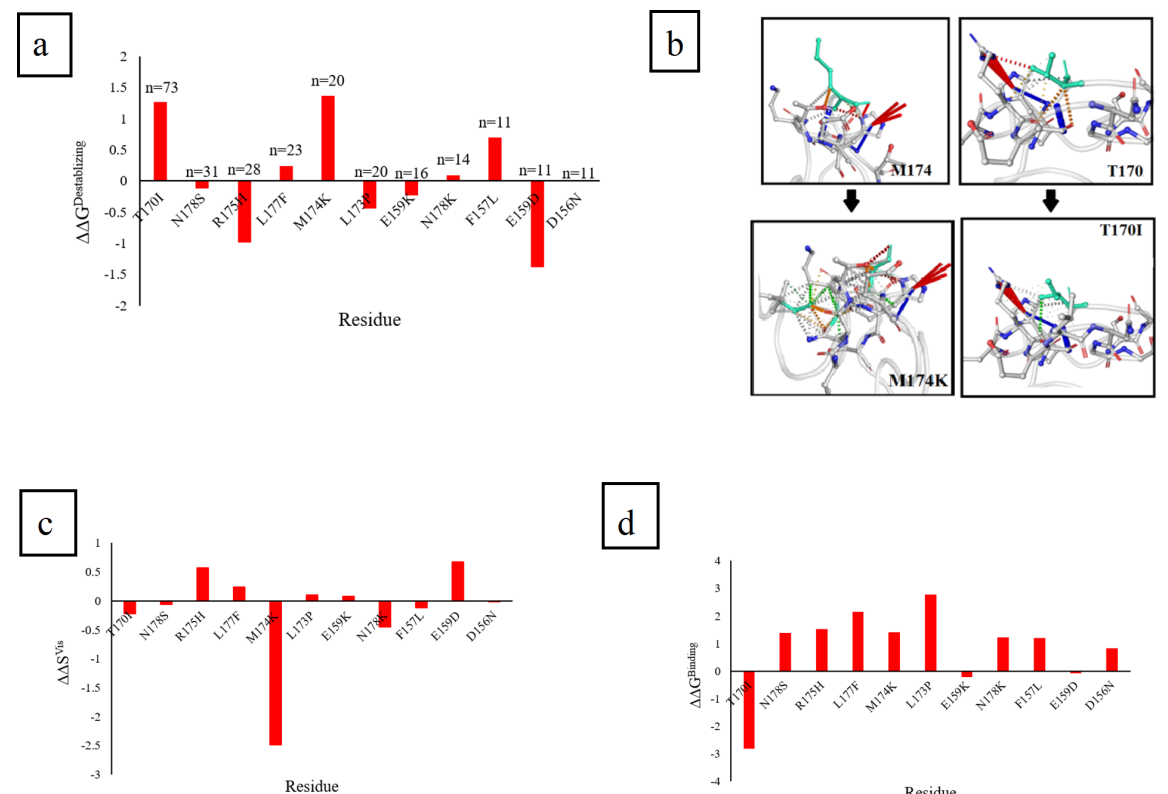

d

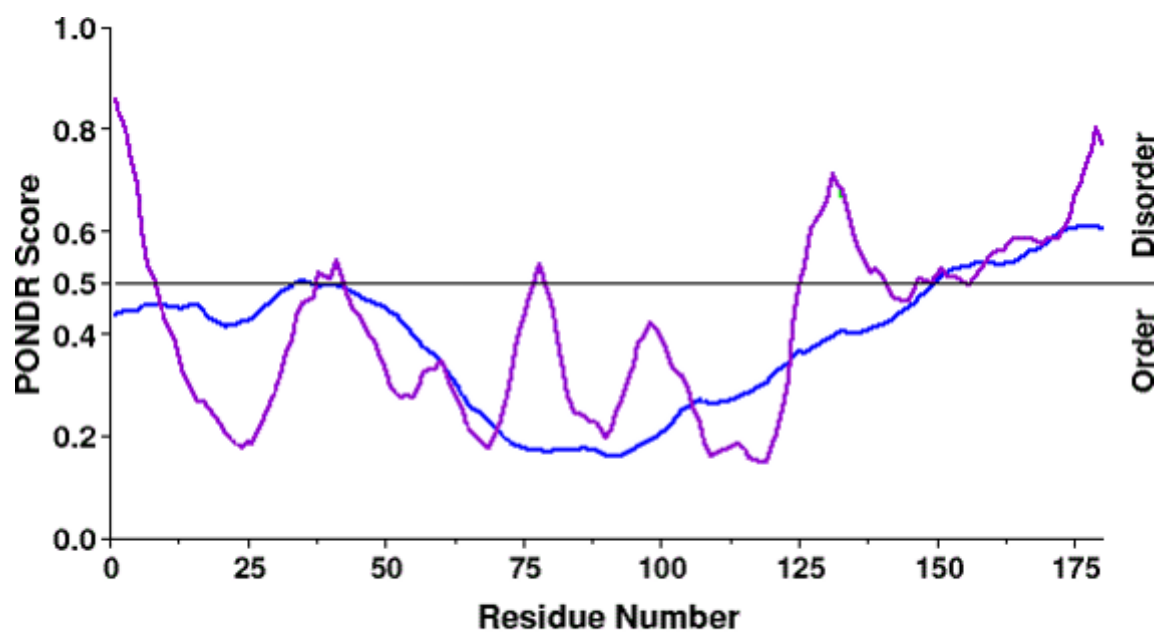

二 vsL2 
a

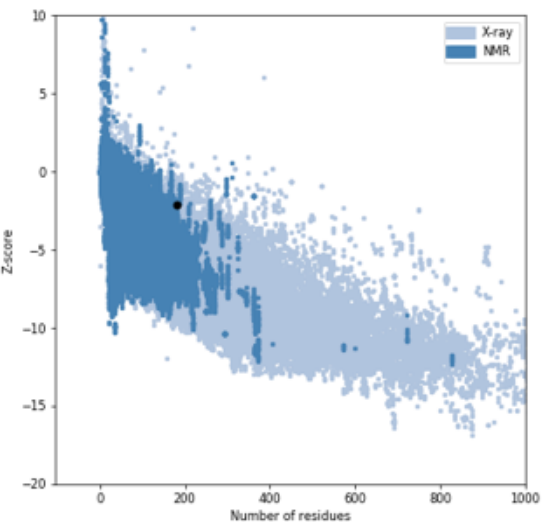

b

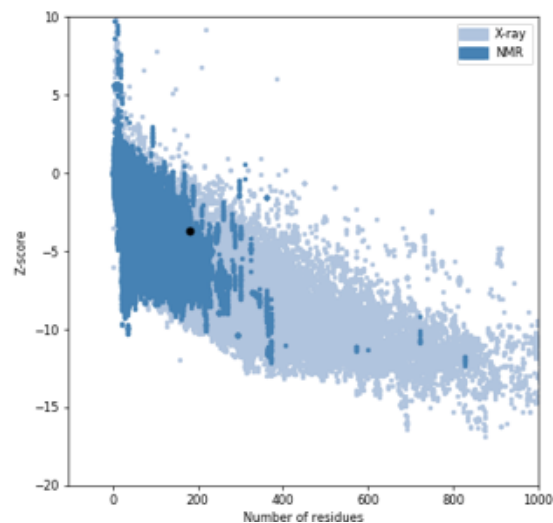

